\title{
Evaluation of Log Odds of Positive Lymph Nodes in Predicting the Survival of Neoadjuvant Therapy Patients with Non-Small Cell Lung Cancer After Surgery: A SEER Cohort-Based Study
}

\section{Qing Wang}

Shanghai Jiao Tong University School of Medicine

\section{Suyu Wang}

Navy Medical University

\section{Zhiyong Sun}

Shanghai Jiao Tong University School of Medicine

\section{Min Cao}

Shanghai Jiao Tong University School of Medicine

Xiaojing Zhao ( $\nabla$ zhaoxiaojing@renji.com )

Shanghai Jiao Tong University School of Medicine

\section{Research Article}

Keywords: log odds of positive lymph nodes (LODDS), non-small cell lung cancer (NSCLC), prognosis, Surveillance, Epidemiology, and End Results (SEER), neoadjuvant therapy

Posted Date: January 12th, 2022

DOl: https://doi.org/10.21203/rs.3.rs-1107770/v1

License: (c) (i) This work is licensed under a Creative Commons Attribution 4.0 International License.

Read Full License 


\section{Abstract \\ Background}

log odds of positive lymph nodes (LODDS) is a novel lymph node (LN) descriptor, demonstrating promising prognostic value in many tumors. However, there was limited information on LODDS in nonsmall cell lung cancer (NSCLC) patients, especially those receiving neoadjuvant therapy followed by lung surgery.

\section{Methods}

A total of 2,059 NSCLC patients who received neoadjuvant therapy and surgery were identified in the Surveillance, Epidemiology, and End Results (SEER) database. We used the X-tile software to calculate the cut-off value of LODDS. Kaplan-Meier survival analysis and receiver operating characteristics (ROC) curve were used to compare the predictive value of the American Joint Committee on Cancer (AJCC) N staging descriptor and LODDS. Univariate and multivariate Cox regression and inverse probability of treatment weighting (IPTW) analyses were conducted to construct the model predicting the prognosis.

\section{Results}

LODDS showed better differentiating ability in survival analysis than $\mathrm{N}$ staging descriptor (Log-rank test, $P<0.0001$ vs. $P=0.031)$. The ROC curve demonstrated that the AUC of LODDS was significantly higher than the $\mathrm{N}$ staging descriptor in 1-year, 3-year, and 5-year survival analyses (All $\mathrm{P}<0.05)$. Univariate and multivariate Cox regression analysis showed that the LODDS was an independent risk factor for NSCLC patients receiving neoadjuvant therapy followed by surgery, both before and after IPTW (all P<0.001). A clinicopathological model with LODDS, age, gender, T, and radiotherapy could better predict the prognosis.

\section{Conclusions}

Compared with the AJCC N staging descriptor, LODDS exhibits better predictive ability for NSCLC patients receiving neoadjuvant therapy followed by surgery. A multivariate clinicopathological model with LODDS included demonstrates sound performance in predicting the prognosis.

\section{Introduction}

Lung cancer is the most common cause of death from cancer worldwide, causing 69,410 male deaths and 62,470 female deaths in the 2021 year of USA alone(1). As a prominent type of lung cancer, nonsmall cell lung cancer (NSCLC) accounts for about $85 \%$ of all types of lung cancer cases, with $60 \%$ for lung adenocarcinoma and $15 \%$ for lung squamous cell carcinoma (SCC) as the most common 
histological subtypes(2). With the advent of the new era of targeted therapy and immunotherapy, the overall survival of NSCLC has gained a considerable increase for each stage(3). Despite the novel treatments, lung surgery remains the most substantial and supportive tool for NSCLC. For locally advanced NSCLC patients, neoadjuvant therapy plays a crucial role in downstaging lung cancer and providing an opportunity for surgery, which effectively improves the prognosis(4). Traditional neoadjuvant therapy included chemotherapy and chemoradiation, while molecular-targeted therapy and immunotherapy are evolving as revolutionary neoadjuvant treatments for NSCLC(5). However, there are limited tools and predictive models for predicting the prognosis of patients receiving neoadjuvant therapy followed by lung surgery.

The American Joint Committee on Cancer (AJCC) TNM staging system is the most commonly used tool for recurrence and survival prediction(6). For $\mathrm{N}$ descriptor, the lymph node (LN) is based on the lymphatic region involved, without any information of the number of dissected lymph nodes (NDLN) and the number of positive lymph nodes (NPLN)(7). Log odds of positive lymph nodes (LODDS) is a novel lymph node descriptor, showing advantages over $\mathrm{N}$ stating descriptor of TNM system in many malignancies, including rectal cancer(8), gallbladder cancer(9), gastric adenocarcinoma(10), cervical cancer(11), esophageal carcinoma(12), and so on. Wang et al. reported that the nomogram combining the TNM stage with LODDS+LNR performed better than AJCC 8th TNM stage in clinical practicability(13). In addition, Yu and his colleagues found that LODDS exhibited better predictive power than the N, NPLN, LNR staging system(14). However, there was no previous report on the application of LODDS in predicting the prognosis for patients receiving neoadjuvant therapy followed by lung surgery. In this study, we screened the suitable cases from the SEER database and compared the value of LODDS and TNM N descriptors. At last, we tried to construct a model combining LODDS with clinicopathological characteristics for a better prediction. This study was presented following the TRIPOD reporting checklist(15).

\section{Materials And Methods}

\section{Patient selection}

All patients were selected from the SEER database (http://seer.cancer.gov/). Eighteen population-based cancers were selected in the SEER database, with the SEER*Stat program ( $v$ 8.3.9) used to extract the information of patients with lung cancer. The extraction conditions were as follows: "the location of the disease: lung and bronchus" and "diagnosis year: 2004-2015." In the research, we enrolled patients with primary lung cancer who received neoadjuvant therapy and lung surgery between 2004 and 2015. Figure 1 shows the flowchart of the patient selection. Following variables were extracted: "Age recode with $<1$ year olds", "Race recode (White, Black, Other)", "Sex", "Marital status", "Derived AJCC T, 6th ed (20042015)", "Derived AJCC M, 6th ed (2004-2015)", "Primary Site - labeled", "Histologic Type ICD-0-3", "RX Summ--Surg Prim Site (1998+)", "CS tumor size (2004-2015)", "CS Tumor Size/Ext Eval (2004-2015)", "Grade (thru 2017)", "Survival months", "Vital status recode (study cut-off used)", "Regional nodes positive (1988+)", "Regional nodes examined (1988+)", “CS Reg Node Eval (2004-2015)", "First malignant primary 
indicator". And the AJCC TNM staging system was updated to the 8th version. The exclusion criteria were as follows: (a) patients with metastatic disease; (b) patients without lung surgery performed; (c) patients in whom lung cancer was not the one primary only tumor; (d) patients not receiving neoadjuvant therapy; (e) patients without information about the number of retrieved and positive lymph nodes; (f) patients with unknown race, marital status, tumor site, laterality, grade, $\mathrm{T}$ stage, and $\mathrm{N}$ stage.

\section{Ethical statement}

The informed consent was waived, and the institutional review board sought no ethical approval because SEER is a public database and all SEER data contained no personally-identifying information. We conducted this study following the Declaration of Helsinki and the Harmonized Tripartite Guideline for Good Clinical Practice from the International Conference on Harmonization.

\section{LODDS calculation}

LODDS was calculated by the formula of $\operatorname{In}([N P L N+0.5] /[N D L N-N P L N+0.5])$, when NPLN meant the number of positive lymph nodes and NDLN meant the number of dissected lymph nodes. X-Tile software (version 3.6.1) was used to produce the optimal cut-off of LODDS with the maximal survival difference between different groups(16). LODDS was divided into three ranges: LODDS $<-1.07,-1.07 £ L O D D S<-0.27$, and LODDS ${ }^{3}-0.27$.

\section{Statistical analysis}

R software (version 4.0.2) was used to in statistical analysis. A P value of less than 0.05 was considered statistically significant. Categorical variables were presented as proportions. Chi-square tests or Fisher's precision probability test were performed in different evaluations of categorical variables.Univariate and multivariate Cox regression analysis was conducted to screen the risk factors for the overall survival when the variables with $P$ value less than 0.05 were finally incorporated into the risk model.

A Kaplan-Meier survival curve and the log-rank test were conducted to compare the overall survival of patients with different LODDS ranges and different $\mathrm{N}$ classifications. Receiver operating characteristics (ROC) was used to evaluate the predictive value of $\mathrm{N}$ classification, LODDS, and the multivariate model for the patients' long-term outcomes. Weighted mean rank (WMR) statistics were used to compare the area under the curve (AUC) of N classification, LODDS, and the multivariate model(17). To better balance the baseline of patients of different LODDS ranges, propensity scores were calculated using generalized boosted models, and inverse probability of treatment weighting (IPTW) was used to adjust the Cox regression analyses(18). In addition, prediction accuracy was compared by calculating the integrated discrimination improvement (IDI) and the net reclassification improvement (NRI) between LODDS, $\mathrm{N}$ classification, and multivariate model.

\section{Results}


In table 1, we compared the demographical and clinicopathological characteristics of patients with different LODDS ranges. A total of 2059 patients in the SEER database were enrolled in this study and divided into three groups, patients with LODDS $<-1.07,-1.07 £$ LODDS $<-0.27$, and LODDS $3-0.27$. There was no difference between different groups in age, marital status, surgery type, and radiotherapy (all $P>0.05$ ). However, the variables of gender, race, laterality, primary site, histology type, differentiation, and chemotherapy were significantly different among the three groups (All $\mathrm{P}<0.05)$. The patients with LODDS ${ }^{3}-0.27$ had higher proportions of female, right laterality, the primary site of lower lobe, adenocarcinoma, low differentiation grade, low T1 stage, and chemotherapy. Since LODDS was calculated with the NDLN and the NPLN, the patients with LODDS $3-0.27$ had a higher $\mathrm{N}$ stage, more regional nodes examined and positive. We conducted the IPTW to eliminate the demographical and clinicopathological characteristics of patients with different LODDS ranges. As shown in Figure $\mathrm{S} 1$, the absolute standardized differences of variables were decreased under 0.2 and mostly under 0.1 , indicating that the three groups were well matched after IPTW.

\section{Univariate and multivariate Cox regression analysis}

We conducted the univariate and multivariate Cox regression analysis to confirm the independent risk factors for the patients' survival, shown in Table 2 and Table 3. Before IPTW, the univariate analysis demonstrated that LODDS, age, gender, $\mathrm{T}$ stage, $\mathrm{N}$ stage, and radiotherapy were significantly associated with the OS of the patients (All $\mathrm{P}<0.05$ ). However, the multivariate analysis showed that LODDS, age, gender, $T$ stage, and radiotherapy were independent risk factors for patients' survival (All $P<0.05)$, with $N$ stage excluded.

After IPTW, the univariate analysis results were similar to the previous results, showing that LODDS, age, gender, $\mathrm{T}$ stage, $\mathrm{N}$ stage, and radiotherapy were variables with statistical significance, while the race, marital status, primary site, histologic type, differentiation, and surgery type were newly added variables (All $\mathrm{P}<0.05$ ). Furthermore, the multivariate regression analysis indicated that LODDS, age, gender, race, marital status, primary site, differentiation, and T stage were independent risk factors for patients' survival (All P<0.05), with $\mathrm{N}$ stage excluded. Whether with or without IPTW, the LODDS was an independent risk factor for the prognosis of patients receiving neoadjuvant therapy followed by lung surgery.

We also conducted a subgroup analysis to validate the significance of LODDS further. By dividing the patients into different subgroups through the variable, we further compared the relative risk of different LODDS ranges. We found that the higher LODDS was associated with higher risks in most subgroups, as shown in Table 4. However, in subgroups of middle lobe, overlapping primary site, grade I differentiation, grade IV differentiation, and N3 stage, there was no statistical significance among different LODDS ranges, which could be caused by the relatively lower sample size.

\section{Survival analysis}


We compared the long-term survival of patients with different $\mathrm{N}$ classifications, shown in Figure 2A. Although the patients with different $\mathrm{N}$ stages presented different survival curves with a P value of 0.036 , the curve was not separate and mostly overlapped. Nevertheless, when we divided the patients into three groups of LODDS ranges, we found that the curve was much more separate (Figure 2B). The patients with LODDS $<-1.07$ demonstrated the best survival status than the other two groups, while the middle group $(-1.07 £ L O D D S<-0.27)$ had better overall survival than the patients with LODDS $3-0.27(P<0.0001)$. Even after IPTW, the survival curve was still significant among three groups $(P<0.0001)$, shown in Figure 3 .

\section{ROC analysis}

In addition, we compared the accuracy and prognostic value of $\mathrm{N}$ classification, LODDS, and a multivariate model by ROC curve and AUC comparison. We used a multivariate model with five variables which had been shown to be independent prognostic indicatiors in multivariate analysis in Table 2: LODDS, age, gender, T stage, and radiotherapy. As shown in Figure 4, LODDS had significantly higher AUC than $\mathrm{N}$ classification in 1-year $(P=0.008)$, 3-year $(P=0.007), 5$-year OS $(P=0.010)$, but not in 10-year OS $(P=0.228)$. However, the multivariate model had significantly higher AUC than LODDS and N classification in 1-year, 3-year, 5-year, and 10-year OS (All P<0.001). We also compared the IDI and NRI of $N$ classification, LODDS, and multivariate model, as shown in Table 5. When listing the LODDS as the reference, we found the IDI and NRI of $\mathrm{N}$ classification were negative. At the same time, those of the multivariate model was positive, suggesting that the LODDS had significantly higher predictive accuracy than $\mathrm{N}$ classification but was lower than the multivariate model $(\mathrm{P}<0.05)$.

\section{Discussion}

Controversies over the nodal status of the 8th TNM staging system have existed for many years. In summary, there are four commonly used nodal classifications in lung cancer, including $\mathrm{N}$ classification, NPLN, LNR, and LODDS(19). The N classification in the TNM staging system is the most commonly used prognostic tool for patients with lung cancer. The $\mathrm{N}$ classification is easy to understand and remember in lung cancer, which categorizes no metastasis to lymph node as N0, metastasis to ipsilateral peribronchial and/or hilar nodes and intrapulmonary nodes as N1, metastasis to ipsilateral mediastinal and/or subcarinal nodes as N2, metastasis to Contralateral mediastinal and/or hilar, as well as any supraclavicular lymph nodes as N3(20). The TNM staging system helps clinicians to decide the treatment and predict the prognosis. However, the $\mathrm{N}$ classification is based on the anatomic position of the positive nodes, without any quantity information, leading to inaccuracy and low discrimination power(21). In this study, we found that the AUC of N classification was only $0.49395 \% \mathrm{Cl}(0.461-0.526), 0.53895 \% \mathrm{Cl}$ (0.513-0.563), $0.54995 \% \mathrm{Cl}(0.522-0.577)$, and $0.60395 \% \mathrm{Cl}(0.554-0.651)$ in 1-year, 3-year, 5-year, and 10-year survival respectively. The low discriminative power of TNM staging $\mathrm{N}$ classification calls for a more accurate nodal status assessment tool.

For patients receiving radical lung cancer resection, systematic lymph node dissection (SND) is the standard procedure in surgical treatment for NSCLC(22), especially for patients receiving neoadjuvant 
therapy, who were usually diagnosed with stage II-III, when systematic lymph node dissection is a must. In this study, $78.1 \%$ of patients received lobectomy, and $19.3 \%$ received pneumonectomy, with only $2.7 \%$ of patients having sublobectomy. Mun reported that lobe-specific mediastinal lymph node dissection (MLND) is vital for $\mathrm{pN} 1$ patients, while SND contributes to survival for $\mathrm{pN2}$ patients after recurrence(23). The lymph nodes retrieved in the surgery provide sufficient knowledge about the nodal status with quantity information. NPLN stands for the number of positive lymph nodes, requiring retrieval of the lymph nodes from the surgery(14). However, the NPLN can be significantly affected by the surgical technique and the number of examined lymph nodes because the pathological results are dependent on the lymph node dissection. Using a Chinese multi-institutional registry and the US SEER database on stage I to IIIA resected NSCLC, Liang recommended 16 examined lymph nodes for prognostic stratification(24).

Ratio-based nodal evaluation methods are also used, not requiring the number of examined lymph nodes, including LNR and LODDS. LNR is calculated by dividing the NPLN with NDLN. LODDS is calculated using the formula: $\log (\mathrm{NPLN}+0.50) /(\mathrm{NDLN}-\mathrm{NPLN}+0.50)$, so LODDS is the only indicator with the numbers of dissected, positive, and negative LNs included. The controversy over the comparison between LNR and LODDS consists since they demonstrate advantages against each other in different situations $(25,26)$. However, the LODDS shows superiority over LNR for lung cancer in most studies. Yu and his colleagues demonstrated that LODDS showed better predictive performance than N, NPLN, and LNR among patients with node-positive SCC after surgery(14). Deng found that LODDS and LNR performed slightly differently in patients with different resected LNs. He proved that LODDS was slightly better than LNR for patients with $<10$ resected LNs, while LNR was slightly better than LODDS for patients with $\geq 10$ resected LNs(27). When combined, LODDS and LNR had the highest prediction accuracy than other models for cancer-specific survival and overall survival of lung adenocarcinoma patients after surgery(13). However, there is no previous report on the predictive ability and accuracy of LODDS in patients receiving neoadjuvant therapy and surgery. In this study, we found that LODDS could effectively differentiate the patient's prognosis. Also, LODDS demonstrated a much higher AUC than N classification 1-year, 3-year, and 5-year prediction, but not in 10-year prediction. The univariate and multivariate Cox regression analysis demonstrated that LODDS was an independent risk factor for patients' overall survival. The subgroups analysis confirmed the results in different subgroups.

We noticed that the baseline characteristics and demographical data of patients with different LODDS ranges were significantly different. To eliminate the bias caused by the difference, we applied the IPTW to balance the baseline characteristics and demographical data. Whether before or after IPTW, the LODDS showed statistical significance in the Kaplan-Meier curve and regression analysis. Due to its excellent predictive ability, LODDS was incorporated into the multivariate model to construct a nomogram. Wang's nomogram included LODDS+LNR as the nodal status factor, which showed an excellent predictive ability with a high C-index ( 0.7222 for nomogram for CSS, 0.6920 for nomogram for OS) for patients with T14N0-2M0 lung adenocarcinoma after surgery(13). This study applied a multivariate model with five critical factors: LODDS, age, gender, T stage, and radiotherapy. This model showed higher AUC than $\mathrm{N}$ classification and LODDS. The multivariate model's predictive performance indicator IDI and NRI were 
also higher than $\mathrm{N}$ classification and LODDS, which proved that LODDS is an independent and compatible factor for lymph node staging and could be incorporated into the risk assessment model well.

Several limitations of this study must be noted. On the one hand, many important data are absent from the SEER database, including smoking history, sequence of surgery and chemotherapy, novel treatments of tyrosine kinase inhibitors treatment, and immune checkpoint inhibitors. The missing data might lead to the worse predictive effect of the nomogram. We tried to construct a nomogram based on our findings but failed in this study because the $\mathrm{C}$-index was very low. We suspected that the low $\mathrm{C}$-index of the nomogram was caused by the heterogeneity of the patients, who received very different treatment regimens. On the other hand, the new era of tyrosine kinase inhibitors and immune checkpoint inhibitors bring the paradigm-shifting for the neoadjuvant therapy for NSCLC patients, which challenges the LODDS and other nodal status indicators.

\section{Conclusions}

For NSCLC patients receiving neoadjuvant therapy followed by surgery, LODDS presents better predictive ability than the AJCC N staging descriptor. A multivariate clinicopathological model with LODDS included demonstrates exemplary performance in predicting the prognosis. LODDS provides clinicians with more accurate nodal status information, while the nomogram and external validation are required in future studies.

\section{Abbreviations}

AIC Akaike information criterion

AJCC American Joint Committee on Cancer

$\mathrm{Cl}$ confidence interval

C-index Harrell concordance index

HR hazard ratio

ICD-0-3 3rd edition of the International Classification of Diseases for Oncology

IDI integrated discrimination improvement

IQR interquartile range

IPTW inverse probability of treatment weighting

KM Kaplan-Meier

LNR lymph node ratio 
LODDS log odds of positive lymph nodes

LR likelihood ratio

NDLN number of dissected lymph nodes

NPLN number of positive lymph nodes

NRI net reclassification improvement

NSCLC non-small cell lung cancer

OS overall survival

PRCDA purchased/referred care delivery areas

ROC receiver operating characteristics

SEER Surveillance, Epidemiology, and End Results

SCC squamous cell carcinoma

TNM tumor, node, metastasis

\section{Declarations}

\section{Ethics approval and consent to participate}

The study was conducted in accordance with the Declaration of Helsinki (as revised in 2013) and the Harmonized Tripartite Guideline for Good Clinical Practice from the International Conference on Harmonization. No approval by the institutional review board was sought, and no individual patient consent was required, because SEER is a public database and the data are de-identified. The authors confirm that they are accountable for all aspects of the work (if applied, including full data access, integrity of the data and the accuracy of the data analysis) in ensuring that questions related to the accuracy or integrity of any part of the work are appropriately investigated and resolved.

\section{Consent for publication}

Not applicable.

\section{Availability of data and materials}

The dataset supporting the conclusions of this article is available in the SEER ${ }^{\star}$ Stat software (version 8.3.8; RRID:SCR_003293; https://seer.cancer.gov/resources/). The primary data can be accessed through SEER ${ }^{\star}$ Stat software with certain filter according to the methods in the manuscript. 


\section{Competing interests}

All authors have completed the ICMJE uniform disclosure form. The authors have no conflicts of interest to declare.

\section{Funding}

None.

\section{Authors' contributions}

QW, SW, and XZ conceived and designed the analysis, collected the data, performed the analysis and wrote the paper. ZS and QWconceived and designed the analysis, contributed data or analysis tools, performed the analysis. MC and SW interpreted the data and wrote the paper. QW and SW conceived and designed the analysis and interpreted the data. All authors read and approved the final manuscript.

\section{Acknowledgements}

The authors would like to thank all patients and staff who have participated in the SEER program.

\section{References}

1. Siegel RL, Miller KD, Fuchs HE, et al. Cancer Statistics, 2021. CA Cancer J Clin 2021;71:7-33.

2. Duma N, Santana-Davila R, Molina JR. Non-Small Cell Lung Cancer: Epidemiology, Screening, Diagnosis, and Treatment. Mayo Clin Proc 2019;94:1623-40.

3. Herbst RS, Morgensztern D, Boshoff C. The biology and management of non-small cell lung cancer. Nature 2018;553:446-54.

4. Blumenthal GM, Bunn PA, Jr., Chaft JE, et al. Current Status and Future Perspectives on Neoadjuvant Therapy in Lung Cancer. J Thorac Oncol 2018;13:1818-31.

5. Zheng Y, Jaklitsch MT, Bueno R. Neoadjuvant Therapy in Non-Small Cell Lung Cancer. Surg Oncol Clin N Am 2016;25:567-84.

6. Lim W, Ridge CA, Nicholson AG, et al. The 8(th) lung cancer TNM classification and clinical staging system: review of the changes and clinical implications. Quant Imaging Med Surg 2018;8:709-18.

7. Hattori A, Takamochi K, Oh S, et al. New revisions and current issues in the eighth edition of the TNM classification for non-small cell lung cancer. Jpn J Clin Oncol 2019;49:3-11.

8. Huang $\mathrm{B}, \mathrm{Ni} \mathrm{M}, \mathrm{Chen} \mathrm{C}$, et al. LODDS is superior to lymph node ratio for the prognosis of nodepositive rectal cancer patients treated with preoperative radiotherapy. Tumori 2017;103:87-92.

9. Xiao Z, Shi Z, Hu L, et al. A new nomogram from the SEER database for predicting the prognosis of gallbladder cancer patients after surgery. Ann Transl Med 2019;7:738.

10. Aurello P, Petrucciani N, Nigri GR, et al. Log odds of positive lymph nodes (LODDS): what are their role in the prognostic assessment of gastric adenocarcinoma? J Gastrointest Surg 2014;18:1254-60. 
11. Wang C, Yang C, Wang W, et al. A Prognostic Nomogram for Cervical Cancer after Surgery from SEER Database. J Cancer 2018;9:3923-8.

12. Ye G, Chen Z, Wang L, et al. Log odds of positive lymph nodes predicts survival in patients treated with neoadjuvant therapy followed by esophagectomy. J Surg Oncol 2020;121:1074-83.

13. Wang $\mathrm{S}, \mathrm{Yu} \mathrm{Y}, \mathrm{Xu}$ W, et al. Dynamic nomograms combining $\mathrm{N}$ classification with ratio-based nodal classifications to predict long-term survival for patients with lung adenocarcinoma after surgery: a SEER population-based study. BMC Cancer 2021;21:653.

14. Yu Y, Zhang P, Yao R, et al. Prognostic value of log odds of positive lymph nodes in node-positive lung squamous cell carcinoma patients after surgery: a SEER population-based study. Transl Lung Cancer Res 2020;9:1285-301.

15. Moons KG, Altman DG, Reitsma JB, et al. Transparent Reporting of a multivariable prediction model for Individual Prognosis or Diagnosis (TRIPOD): explanation and elaboration. Ann Intern Med 2015;162:W1-73.

16. Camp RL, Dolled-Filhart M, Rimm DL. X-tile: a new bio-informatics tool for biomarker assessment and outcome-based cut-point optimization. Clin Cancer Res 2004;10:7252-9.

17. Saha-Chaudhuri P, Heagerty PJ. Non-parametric estimation of a time-dependent predictive accuracy curve. Biostatistics 2013;14:42-59.

18. McCaffrey DF, Griffin BA, Almirall D, et al. A tutorial on propensity score estimation for multiple treatments using generalized boosted models. Stat Med 2013;32:3388-414.

19. Zhao Y, Li G, Zheng D, et al. The prognostic value of lymph node ratio and log odds of positive lymph nodes in patients with lung adenocarcinoma. J Thorac Cardiovasc Surg 2017;153:702-9.e1.

20. Goldstraw P, Chansky K, Crowley J, et al. The IASLC Lung Cancer Staging Project: Proposals for Revision of the TNM Stage Groupings in the Forthcoming (Eighth) Edition of the TNM Classification for Lung Cancer. J Thorac Oncol 2016;11:39-51.

21. Fortea-Sanchis C, Martínez-Ramos D, Escrig-Sos J. The lymph node status as a prognostic factor in colon cancer: comparative population study of classifications using the logarithm of the ratio between metastatic and nonmetastatic nodes (LODDS) versus the pN-TNM classification and ganglion ratio systems. BMC Cancer 2018;18:1208.

22. Adachi H, Sakamaki K, Nishii T, et al. Lobe-Specific Lymph Node Dissection as a Standard Procedure in Surgery for Non-Small Cell Lung Cancer: A Propensity Score Matching Study. J Thorac Oncol 2017;12:85-93.

23. Mun M, Nakao M, Matsuura $Y$, et al. Oncological outcomes after lobe-specific mediastinal lymph node dissection via multiport video-assisted thoracoscopic surgery. Eur J Cardiothorac Surg 2020;58:i92-i9.

24. Liang W, He J, Shen Y, et al. Impact of Examined Lymph Node Count on Precise Staging and LongTerm Survival of Resected Non-Small-Cell Lung Cancer: A Population Study of the US SEER Database and a Chinese Multi-Institutional Registry. J Clin Oncol 2017;35:1162-70. 
25. Baqar AR, Wilkins S, Wang W, et al. Log odds of positive lymph nodes is prognostically equivalent to lymph node ratio in non-metastatic colon cancer. BMC Cancer 2020;20:762.

26. Occhionorelli S, Andreotti D, Vallese P, et al. Evaluation on prognostic efficacy of lymph nodes ratio (LNR) and log odds of positive lymph nodes (LODDS) in complicated colon cancer: the first study in emergency surgery. World J Surg Oncol 2018;16:186.

27. Deng $W, X u T$, Wang $Y$, et al. Log odds of positive lymph nodes may predict survival benefit in patients with node-positive non-small cell lung cancer. Lung Cancer 2018;122:60-6.

\section{Tables}

TABLE 1 Baseline characteristics of NSCLC patients underwent neoadjuvant therapy 
Variables

\begin{tabular}{llll} 
LODDS $<-1.07$ & $-1.07 \leq$ LODDS $<-0.27$ & LODDS $\geq-0.27$ & $P$ \\
\hline$n=866$ & $n=895$ & $n=298$
\end{tabular}

Age

$\leq 60$ years old

\begin{tabular}{lll}
$365(42.1 \%)$ & $405(45.3 \%)$ & $142(47.7 \%)$ \\
\hline $218(25.2 \%)$ & $229(25.6 \%)$ & $70(23.5 \%)$ \\
\hline $283(32.7 \%)$ & $261(29.2 \%)$ & $86(28.9 \%)$
\end{tabular}

Gender

Female

Male

Race

White

Black

Other

Marital status

Married
Unmarried
Seperated/Divorced/Widowed

Laterality

Right

Left

Primary site

\begin{tabular}{|c|c|c|c|c|}
\hline Main bronchus & $24(2.8 \%)$ & $19(2.1 \%)$ & $6(2.0 \%)$ & \\
\hline Upper lobe & $642(74.1 \%)$ & $584(65.3 \%)$ & $181(60.7 \%)$ & \\
\hline Middle lobe & $22(2.5 \%)$ & $41(4.6 \%)$ & $20(6.7 \%)$ & \\
\hline Lower lobe & $162(18.7 \%)$ & $227(25.4 \%)$ & $83(27.9 \%)$ & \\
\hline Overlapping lesion of lung & $16(1.8 \%)$ & $24(2.7 \%)$ & $8(2.7 \%)$ & \\
\hline Histologic type & & & & $<0.001$ \\
\hline Adenocarcinoma & $343(39.6 \%)$ & $451(50.4 \%)$ & $205(68.8 \%)$ & \\
\hline Squamous cell & $365(42.1 \%)$ & $291(32.5 \%)$ & $57(19.1 \%)$ & \\
\hline
\end{tabular}


Other

Differentiation

Grade I
Grade II
Grade III
Grade IV
Unknown

T

T1

\section{T3}

T4

N

N0
N1
N2
N3

Regional nodes examined

Regional nodes positive

Surgery

Sublobectomy
Lobectomy

Pneumonectomy

Radiotherapy

$447(51.6 \%) \quad 178(19.9 \%)$

$91(10.5 \%) \quad 147(16.4 \%)$

$208(24.0 \%) \quad 257(28.7 \%)$

$279(32.2 \%) \quad 270(30.2 \%)$

$288(33.3 \%) \quad 221(24.7 \%)$

$112(12.9 \%) \quad 198(22.1 \%)$

$293(33.8 \%) \quad 502(56.1 \%)$

$14(1.6 \%)$

17 (1.9\%)

$13.0(9.0-20.0)$

$.0(0.0-0.0)$

$9.0(4.0-15.0)$

$1.0(0.0-2.0)$

$23(2.7 \%)$

$676(78.1 \%)$

$167(19.3 \%)$

$.3 \%)$

$291(33.6 \%)$

$575(66.4 \%)$

No/Unknown

Yes

$275(30.7 \%)$
$620(69.3 \%)$

$39(4.4 \%)$

$36(12.1 \%)$

0.032

$9(3.0 \%)$

101 (33.9\%)

138 (46.3\%)

$2(0.7 \%)$

48 (16.1\%)

$<0.001$

58 (19.5\%)

129 (43.3\%)

67 (22.5\%)

$44(14.8 \%)$

Chemotherapy

No/Unknown

Yes

$24(2.8 \%)$

$842(97.2 \%)$

11 (3.7\%)

233 (78.2\%)

$54(18.1 \%)$

$42(14.1 \%)$

$246(82.6 \%)$

10 (3.4\%)

$9.0(5.0-15.0)<0.001$

$5.0(2.0-9.0)<0.001$

0.418

0.082

$80(26.8 \%)$

218 (73.2\%)

0.008

0 (0.0\%)

298 (100.0\%)


Categorical variables are presented with number (percentage) and continuous variables are reported as median with interquartile range. NSCLC, non-small cell lung cancer; LODDS, log odds of positive lymph nodes.

TABLE 2 Cox regression analysis of NSCLC patients underwent neoadjuvant therapy before IPTW 
Variables

Univariable analysis

Multivariable analysis

$\mathrm{HR}(95 \% \mathrm{Cl})$

$\mathrm{P}$

HR $(95 \% \mathrm{Cl})$

$\mathrm{P}$

LODDS

LODDS $<-1.07$

$-1.07 \leq$ LODDS $<-0.27$

LODDS $\geq-0.27$

Age

$\leq 60$ years old

61-67 years old

$\geq 68$ years old

Gender

Female

Male

Race

White

Black

Other

Marital status

Married

Unmarried

Seperated/Divorced/Widowed

Laterality

Right

Left

Primary site
Main bronchus

Upper lobe

Middle lobe

Lower lobe

Upper lobe
Middle lobe
Lower lobe

Reference

$1.387(1.220-1.578) \quad<0.001$

$2.026(1.719-2.388) \quad<0.001$

Reference

$1.300(1.123-1.504) \quad<0.001$

$1.583(1.385-1.808)$
Reference

$1.396(1.220-1.598) \quad<0.001$

$2.116(1.759-2.544) \quad<0.001$
Reference

$1.353(1.168-1.568) \quad<0.001$

$1.716(1.497-1.967) \quad<0.001$

Reference

Reference

$1.233(1.098-1.384) \quad<0.001$

$1.246(1.107-1.401) \quad<0.001$

Reference

$0.874(0.715-1.069) \quad 0.19$

$0.858(0.685-1.075) \quad 0.182$

Reference

$1.106(0.929-1.316) \quad 0.259$

$1.064(0.926-1.222) \quad 0.383$

Reference

Reference

$0.900(0.800-1.012) \quad 0.078$

$0.958(0.848-1.083) \quad 0.495$

Reference

$1.105(0.736-1.659) \quad 0.631 \quad 0.968(0.642-1.460) \quad 0.876$

$1.436(0.887-2.327) \quad 0.141 \quad 1.204(0.736-1.967) \quad 0.46$

$1.423(0.939-2.158) \quad 0.096$
Reference

$1.223(0.803-1.861) \quad 0.348$


Overlapping lesion of lung

$1.826(1.083-3.079) \quad 0.024 \quad 1.383(0.814-2.348)$

0.231

Histologic type

Adenocarcinoma

Reference

Squamous cell

$0.986(0.867-1.121) \quad 0.825$

Other

$0.935(0.796-1.098) \quad 0.411$

Differentiation

\begin{tabular}{lllll} 
Grade I & Reference & & Reference & \\
\hline Grade II & $1.266(0.888-1.803)$ & 0.192 & $1.229(0.861-1.753)$ & 0.256 \\
\hline Grade III & $1.403(0.993-1.983)$ & 0.055 & $1.358(0.959-1.923)$ & 0.085 \\
\hline Grade IV & $1.342(0.834-2.160)$ & 0.225 & $1.431(0.886-2.310)$ & 0.143 \\
\hline Unknown & $1.099(0.764-1.580)$ & 0.611 & $1.091(0.757-1.574)$ & 0.639 \\
\hline T & & & & \\
\hline T1 & Reference & & Reference & \\
\hline T2 & $1.153(0.954-1.392)$ & 0.14 & $1.147(0.948-1.389)$ & 0.158 \\
\hline T3 & $1.277(1.061-1.537)$ & 0.01 & $1.363(1.128-1.647)$ & 0.001 \\
\hline T4 & $1.175(0.969-1.424)$ & 0.101 & $1.336(1.094-1.632)$ & 0.004 \\
\hline N & & & & \\
\hline N0 & Reference & & Reference & \\
\hline N1 & $1.132(0.951-1.348)$ & 0.163 & $0.934(0.778-1.120)$ & 0.459 \\
\hline N2 & $1.223(1.069-1.398)$ & 0.003 & $1.021(0.878-1.186)$ & 0.791 \\
\hline N3 & $1.240(0.819-1.877)$ & 0.309 & $0.962(0.631-1.468)$ & 0.858 \\
\hline
\end{tabular}

Surgery

Sublobectomy

Reference

Lobectomy

$0.866(0.648-1.159) \quad 0.334$

Pneumonectomy

$1.086(0.796-1.482) \quad 0.602$

Radiotherapy

No/Unknown

Reference

Reference

Yes

$1.173(1.035-1.330) \quad 0.013$

$1.203(1.054-1.372) \quad 0.006$

Chemotherapy 
No/Unknown Reference

$\begin{array}{lll}\text { Yes } & 0.927(0.619-1.389) & 0.715\end{array}$

NSCLC, non-small cell lung cancer; IPTW, inverse probability of treatment weighting; $\mathrm{HR}$, hazard ratio; $\mathrm{Cl}$, confidence interval; LODDS, log odds of positive lymph nodes.

TABLE 3 Cox regression analysis of NSCLC patients underwent neoadjuvant therapy after IPTW 
Variables

Univariable analysis

Multivariable analysis

$\mathrm{HR}(95 \% \mathrm{Cl})$

$\mathrm{P}$

HR $(95 \% \mathrm{Cl})$

P

LODDS

\begin{tabular}{lllll}
\hline LODDS $<-1.07$ & Reference & & Reference & \\
\hline$-1.07 \leq$ LODDS<-0.27 & $1.445(1.325-1.577)$ & $<0.001$ & $1.437(1.313-1.573)$ & $<0.001$ \\
\hline LODDS $\geq-0.27$ & $2.318(2.127-2.527)$ & $<0.001$ & $2.459(2.227-2.715)$ & $<0.001$ \\
\hline Age & & & & \\
\hline$\leq 60$ years old & Reference & & Reference & \\
\hline $61-67$ years old & $1.282(1.177-1.397)$ & $<0.001$ & $1.360(1.245-1.485)$ & $<0.001$ \\
\hline$\geq 68$ years old & $1.591(1.471-1.721)$ & $<0.001$ & $1.803(1.659-1.959)$ & $<0.001$ \\
\hline Gender & & & & \\
\hline Female & Reference & & Reference & \\
\hline Male & $1.318(1.231-1.411)$ & $<0.001$ & $1.321(1.227-1.422)$ & $<0.001$ \\
\hline Race & & & & \\
\hline White & Reference & & Reference & \\
\hline Black & $0.919(0.814-1.037)$ & 0.169 & $0.989(0.874-1.119)$ & 0.86 \\
\hline Other & $0.827(0.723-0.946)$ & 0.006 & $0.798(0.694-0.917)$ & 0.001 \\
\hline Marital status & & & & $<0.001$ \\
\hline Married & $1.002(0.935-1.073)$ & 0.961 & & \\
\hline Unmarried & Reference & & Reference & \\
\hline Seperated/Divorced/Widowed & $1.064(0.981-1.155)$ & 0.135 & $1.163(1.067-1.269)$ & $<$ \\
\hline Laterality & & & & \\
\hline Right & $1.194(1.078-1.324)$ & $<0.001$ & $1.417(1.272-1.578)$ & $<0.001$ \\
\hline Left & & & & \\
\hline Peference & & & & \\
\hline
\end{tabular}

Primary site

Main bronchus

Reference

Reference

Upper lobe

$1.068(0.833-1.370) \quad 0.603$

$1.109(0.857-1.434) \quad 0.432$

Middle lobe

$1.130(0.842-1.516) \quad 0.416$

$1.265(0.934-1.714) \quad 0.128$

Lower lobe

$1.293(1.002-1.668) \quad 0.048$

$1.373(1.056-1.785) \quad 0.018$ 
Overlapping lesion of lung

$1.498(1.079-2.078) \quad 0.016$

$1.233(0.885-1.719)$

0.216

Histologic type

Adenocarcinoma

Squamous cell

Other

Differentiation

\begin{tabular}{lllll} 
Grade I & Reference & & Reference & \\
Grade II & $1.254(1.015-1.550)$ & 0.036 & $1.223(0.986-1.516)$ & 0.067 \\
\hline Grade III & $1.361(1.106-1.674)$ & 0.004 & $1.258(1.018-1.554)$ & 0.034 \\
\hline Grade IV & $1.153(0.843-1.577)$ & 0.373 & $1.289(0.936-1.776)$ & 0.12 \\
\hline Unknown & $1.116(0.899-1.386)$ & 0.321 & $1.140(0.913-1.423)$ & 0.247 \\
\hline T & & & & \\
\hline T1 & Reference & & Reference & \\
\hline T2 & $1.163(1.038-1.302)$ & 0.009 & $1.116(0.993-1.253)$ & 0.065 \\
\hline T3 & $1.337(1.196-1.494)$ & $<0.001$ & $1.337(1.194-1.497)$ & $<0.001$ \\
\hline T4 & $1.405(1.253-1.575)$ & $<0.001$ & $1.468(1.302-1.656)$ & $<0.001$ \\
\hline N & & & & \\
\hline N0 & Reference & & Reference & \\
\hline N1 & $1.356(1.219-1.508)$ & $<0.001$ & $0.930(0.829-1.043)$ & 0.216 \\
\hline N2 & $1.416(1.299-1.544)$ & $<0.001$ & $1.046(0.947-1.156)$ & 0.377 \\
\hline N3 & $1.542(1.231-1.931)$ & $<0.001$ & $1.062(0.840-1.342)$ & 0.617 \\
\hline
\end{tabular}

Reference Reference

$1.111(1.031-1.197) \quad 0.006 \quad 1.022(0.942-1.109) \quad 0.598$

$\begin{array}{llll}0.982(0.893-1.081) & 0.716 & 0.911(0.822-1.009) & 0.073\end{array}$
Surgery

\begin{tabular}{lllll} 
Sublobectomy & Reference & & Reference & \\
Lobectomy & $0.819(0.687-0.977)$ & 0.027 & $0.913(0.762-1.093)$ & 0.32 \\
\hline Pneumonectomy & $1.028(0.851-1.241)$ & 0.776 & $1.156(0.949-1.408)$ & 0.149 \\
\hline Radiotherapy & & & & \\
\hline No/Unknown & Reference & & Reference & \\
\hline Yes & $1.065(0.989-1.146)$ & 0.096 & $1.067(0.987-1.153)$ & 0.105
\end{tabular}

Chemotherapy 
No/Unknown Reference

Yes $\quad 0.936(0.692-1.266) \quad 0.67$

NSCLC, non-small cell lung cancer; IPTW, inverse probability of treatment weighting; $\mathrm{HR}$, hazard ratio; Cl, confidence interval; LODDS, log odds of positive lymph nodes.

TABLE 4 Multivariable Cox regression analysis of subgroups of NSCLC patients underwent neoadjuvant therapy 
Subgroups

LODDS $<-1.07 \quad-1.07 \leq$ LODDS $<-0.27$

LODDS $\geq-0.27$

HR

$\mathrm{HR}(95 \% \mathrm{Cl})$

$\mathrm{P}$

$\mathrm{HR}(95 \% \mathrm{Cl})$

P

Age

\begin{tabular}{llllll}
$\leq 60$ years old & Reference & $1.649(1.319-$ & $<0.001$ & $2.587(1.924-$ & $<0.001$ \\
& & $2.061)$ & & $3.477)$ & \\
\hline 61-67 years old & Reference & $1.302(0.985-$ & 0.064 & $1.709(1.148-$ & 0.008 \\
& & $1.721)$ & & $2.544)$ & \\
$\geq 68$ years old & Reference & $1.299(1.040-$ & 0.021 & $2.018(1.477-$ & $<0.001$ \\
& & $1.623)$ & & $2.756)$ &
\end{tabular}

Gender

$\begin{array}{llllll}\text { Female } & \text { Reference } & 1.197(0.967- & 0.098 & 1.893(1.449- & <0.001 \\ & & 1.482) & & 2.472) & \\ \text { Male } & \text { Reference } & 1.503(1.261- & <0.001 & 2.252(1.739- & <0.001 \\ & & 1.791) & & 2.917) & \end{array}$

Laterality

$\begin{array}{llllll}\text { Right } & \text { Reference } & 1.260(1.060- & 0.009 & 1.832(1.456- & <0.001 \\ & & 1.498) & & 2.305) & \\ \text { Left } & \text { Reference } & 1.638(1.320- & <0.001 & 2.646(1.936- & <0.001 \\ & & 2.033) & & 3.617) & \end{array}$

Primary site

\begin{tabular}{|c|c|c|c|c|c|}
\hline Main bronchus & Reference & $\begin{array}{l}1.207(0.351- \\
4.149)\end{array}$ & 0.766 & $\begin{array}{l}5.019(0.974- \\
25.856)\end{array}$ & 0.054 \\
\hline Upper lobe & Reference & $\begin{array}{l}1.415(1.202- \\
1.667)\end{array}$ & $<0.001$ & $\begin{array}{l}2.305(1.829- \\
2.905)\end{array}$ & $<0.001$ \\
\hline Middle lobe & Reference & $\begin{array}{l}1.853(0.734- \\
4.676)\end{array}$ & 0.192 & $\begin{array}{l}1.741(0.600- \\
5.053)\end{array}$ & 0.308 \\
\hline Lower lobe & Reference & $\begin{array}{l}1.375(1.037- \\
1.822)\end{array}$ & 0.027 & $\begin{array}{l}1.813(1.244- \\
2.642)\end{array}$ & 0.002 \\
\hline $\begin{array}{l}\text { Overlapping lesion of } \\
\text { lung }\end{array}$ & Reference & $\begin{array}{l}1.684(0.562- \\
5.046)\end{array}$ & 0.352 & $\begin{array}{l}0.745(0.126- \\
4.396)\end{array}$ & 0.746 \\
\hline \multicolumn{6}{|l|}{ Differentiation } \\
\hline Grade I & Reference & $\begin{array}{l}1.723(0.680- \\
4.362)\end{array}$ & 0.251 & $\begin{array}{l}1.940(0.538- \\
6.998)\end{array}$ & 0.311 \\
\hline Grade II & Reference & $\begin{array}{l}1.361(1.030- \\
1.800)\end{array}$ & 0.030 & $\begin{array}{l}2.259(1.592- \\
3.205)\end{array}$ & $<0.001$ \\
\hline Grade III & Reference & $\begin{array}{l}1.301(1.077- \\
1.571)\end{array}$ & 0.006 & $\begin{array}{l}1.971(1.521- \\
2.554)\end{array}$ & $<0.001$ \\
\hline
\end{tabular}




\begin{tabular}{|c|c|c|c|c|c|}
\hline Grade IV & Reference & $\begin{array}{l}0.764(0.338- \\
1.728)\end{array}$ & 0.518 & $\begin{array}{l}0.245(0.018- \\
3.310)\end{array}$ & 0.290 \\
\hline Unknown & Reference & $\begin{array}{l}2.298(1.630- \\
3.239)\end{array}$ & $<0.001$ & $\begin{array}{l}2.923(1.795- \\
4.761)\end{array}$ & $<0.001$ \\
\hline \multicolumn{6}{|l|}{$\mathrm{T}$} \\
\hline T1 & Reference & $\begin{array}{l}1.302(0.855- \\
1.981)\end{array}$ & 0.219 & $\begin{array}{l}1.821(1.071- \\
3.096)\end{array}$ & 0.027 \\
\hline T2 & Reference & $\begin{array}{l}1.582(1.193- \\
2.098)\end{array}$ & 0.001 & $\begin{array}{l}2.003(1.444- \\
2.778)\end{array}$ & $<0.001$ \\
\hline T3 & Reference & $\begin{array}{l}1.468(1.165- \\
1.850)\end{array}$ & 0.001 & $\begin{array}{l}2.115(1.482- \\
3.017)\end{array}$ & $<0.001$ \\
\hline T4 & Reference & $\begin{array}{l}1.184(0.924- \\
1.516)\end{array}$ & 0.182 & $\begin{array}{l}2.291(1.510- \\
3.476)\end{array}$ & $<0.001$ \\
\hline \multicolumn{6}{|l|}{$\mathrm{N}$} \\
\hline NO & Reference & $\begin{array}{l}0.987(0.778- \\
1.251)\end{array}$ & 0.911 & NA & NA \\
\hline N1 & Reference & $\begin{array}{l}1.393(0.993- \\
1.953)\end{array}$ & 0.055 & $\begin{array}{l}1.939(1.221- \\
3.078)\end{array}$ & 0.005 \\
\hline $\mathrm{N} 2$ & Reference & $\begin{array}{l}1.786(1.444- \\
2.209)\end{array}$ & $<0.001$ & $\begin{array}{l}2.523(1.983- \\
3.211)\end{array}$ & $<0.001$ \\
\hline N3 & Reference & $\begin{array}{l}5.538(1.011- \\
30.326)\end{array}$ & 0.049 & $\begin{array}{l}3.885(0.377- \\
40.074)\end{array}$ & 0.254 \\
\hline \multicolumn{6}{|l|}{ Radiotherapy } \\
\hline No/Unknown & Reference & $\begin{array}{l}1.532(1.190- \\
1.972)\end{array}$ & 0.001 & $\begin{array}{l}2.901(2.052- \\
4.101)\end{array}$ & $<0.001$ \\
\hline Yes & Reference & $\begin{array}{l}1.348(1.149- \\
1.582)\end{array}$ & $<0.001$ & $\begin{array}{l}1.884(1.513- \\
2.346)\end{array}$ & $<0.001$ \\
\hline
\end{tabular}

HRs of multivariable analysis of subgroups were adjusted by age, gender, laterality, primary site, differentiation, $\mathrm{T}, \mathrm{N}$, and radiotherapy except for the subgroup variable itself. LODDS, log odds of positive lymph nodes; NSCLC, non-small cell lung cancer; HR: hazard ratio; $\mathrm{Cl}$ : confidence interval.

TABLE 5 Comparison of predictive performance between LODDS and other models 


\begin{tabular}{|c|c|c|c|c|}
\hline Model & IDI (95\%Cl) & $\mathrm{P}$ & NRI $(95 \% \mathrm{Cl})$ & $\mathrm{P}$ \\
\hline \multicolumn{5}{|l|}{ 1-year OS } \\
\hline LODDS & Reference & & Reference & \\
\hline $\mathrm{N}$ classification & $-0.007(-0.014$ to -0.002$)$ & 0.007 & $-0.060(-0.122$ to 0.002$)$ & 0.060 \\
\hline Multivariable model & $0.020(0.012$ to 0.031$)$ & $<0.001$ & $0.184(0.114$ to 0.250$)$ & $<0.001$ \\
\hline \multicolumn{5}{|l|}{ 3-year OS } \\
\hline LODDS & Reference & & Reference & \\
\hline $\mathrm{N}$ classification & $-0.018(-0.030$ to -0.007$)$ & $<0.001$ & $-0.049(-0.157$ to -0.001$)$ & 0.047 \\
\hline Multivariable model & 0.029 (0.017 to 0.045$)$ & $<0.001$ & $0.136(0.096$ to 0.196$)$ & $<0.001$ \\
\hline \multicolumn{5}{|l|}{ 5-year OS } \\
\hline LODDS & Reference & & Reference & \\
\hline $\mathrm{N}$ classification & $-0.025(-0.040$ to -0.012$)$ & $<0.001$ & $-0.074(-0.183$ to -0.025$)$ & 0.007 \\
\hline Multivariable model & $0.036(0.024$ to 0.057$)$ & $<0.001$ & $0.171(0.126$ to 0.224$)$ & $<0.001$ \\
\hline \multicolumn{5}{|l|}{ 10-year OS } \\
\hline LODDS & Reference & & Reference & \\
\hline $\mathrm{N}$ classification & $-0.030(-0.050$ to -0.010$)$ & $<0.001$ & $-0.200(-0.290$ to -0.006$)$ & 0.027 \\
\hline Multivariable model & $0.056(0.034$ to 0.086$)$ & $<0.001$ & $0.258(0.168$ to 0.348$)$ & $<0.001$ \\
\hline
\end{tabular}

Multivariable model includes LODDS, age, gender, T, and radiotherapy. LODDS, log odds of positive lymph nodes; OS, overall survival; IDI, integrated discrimination improvement; NRI, net reclassification improvement; $\mathrm{Cl}$, confidence interval.

\section{Figures}




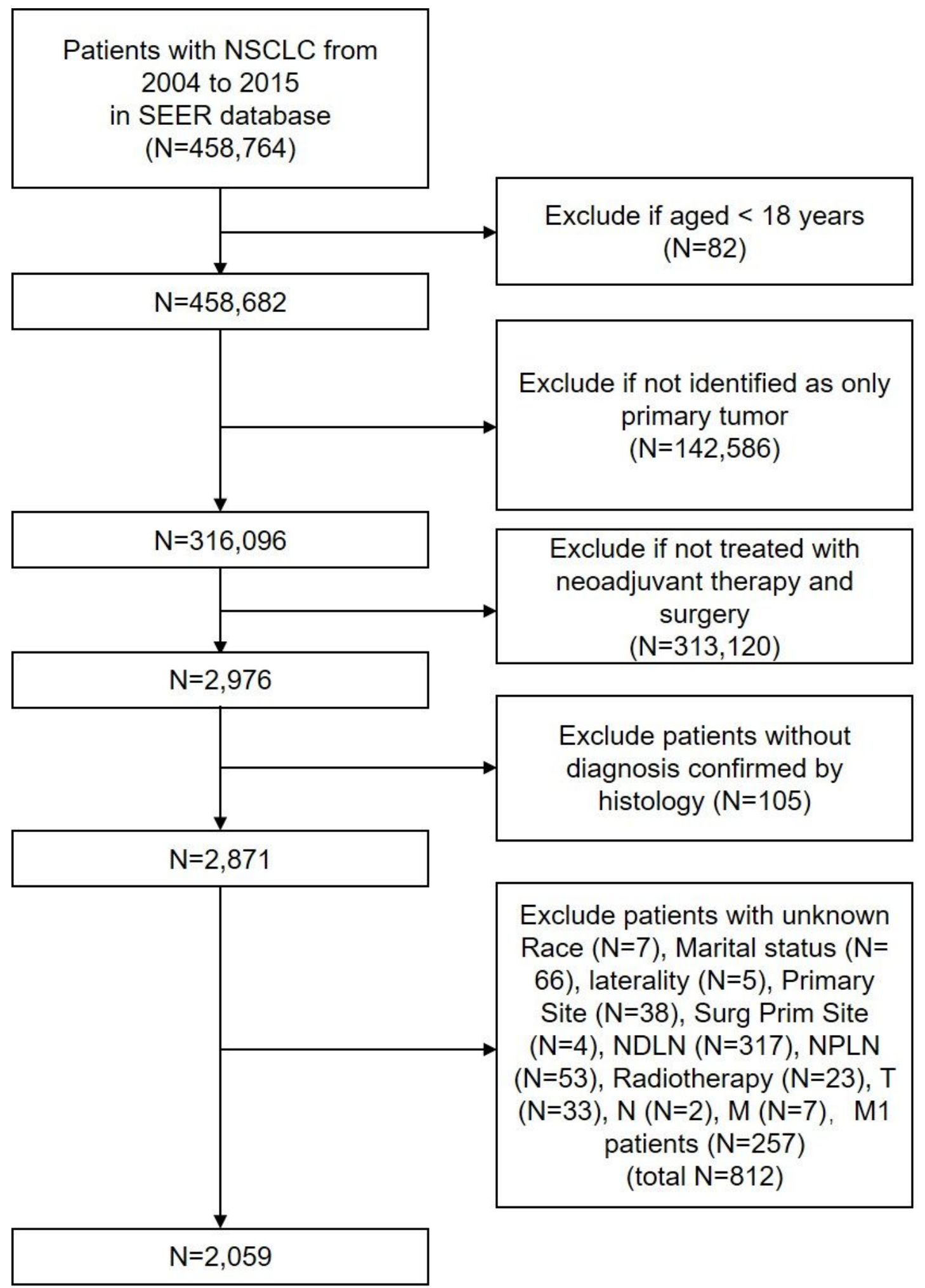

Figure 1

Flowchart of the patient selection 


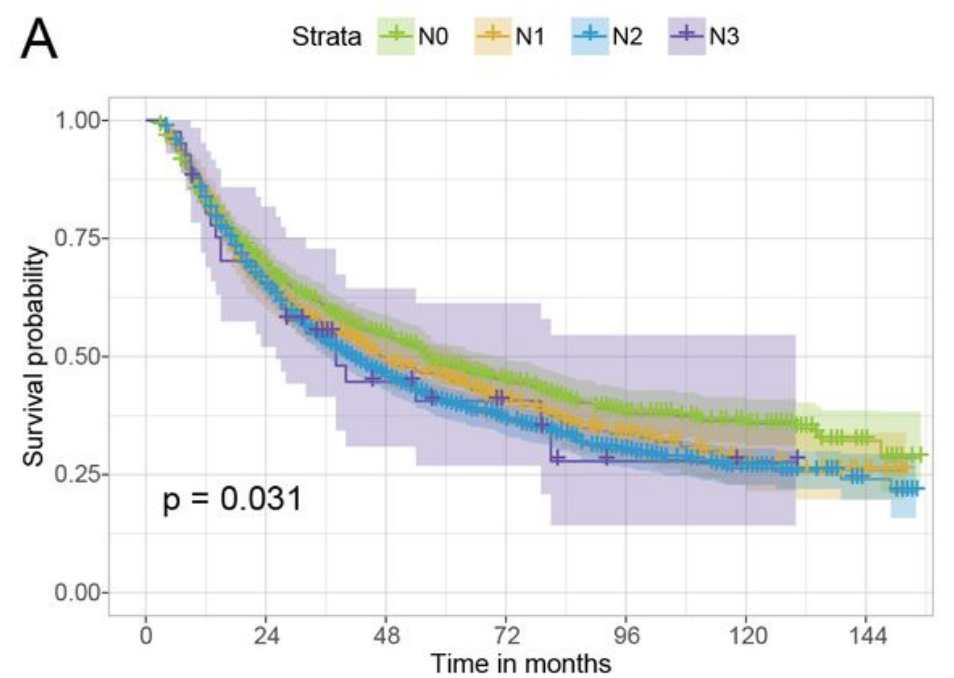

B Strata + LODDS $<-1.07 \pm-1.07 \leq$ LODDS $<-0.27+$ LODDS $\geq-0.27$
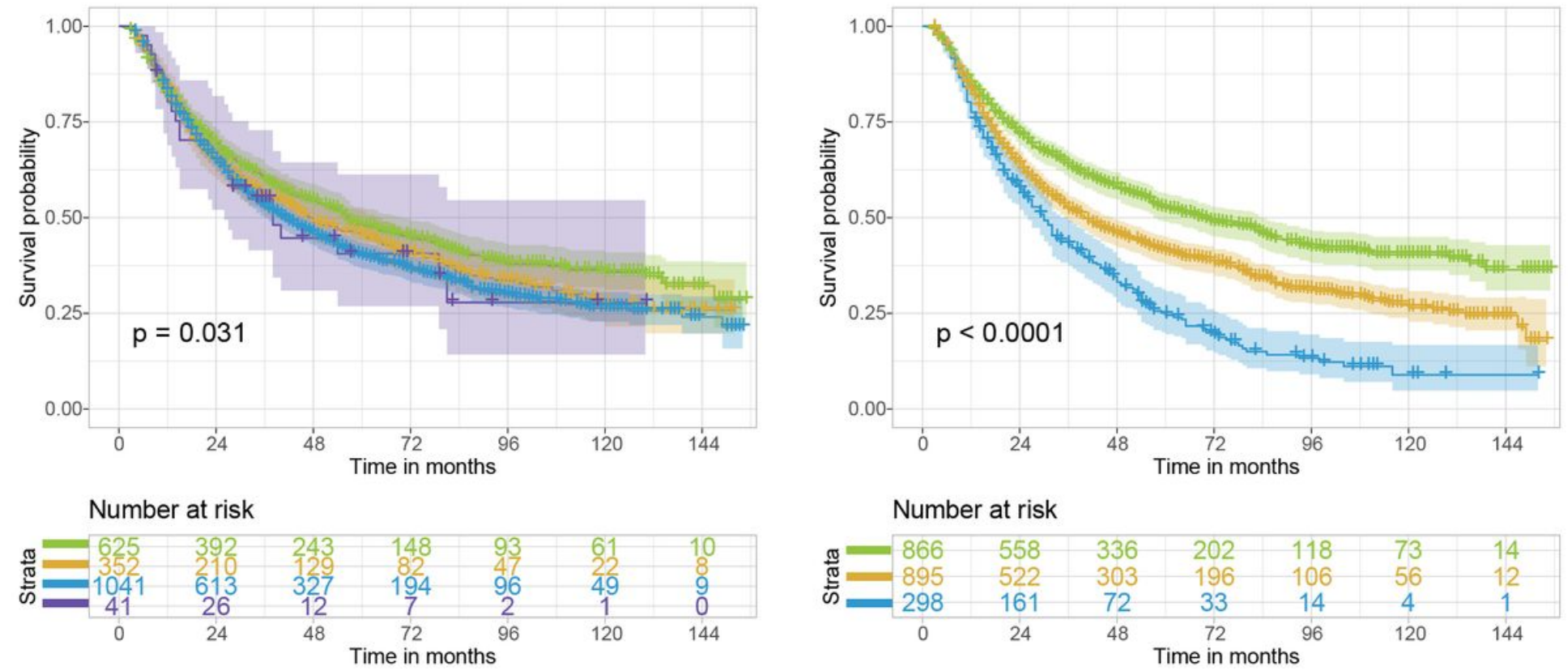

Figure 2

Kaplan-Meier estimates of OS for NSCLC patients who underwent neoadjuvant therapy stratified by $\mathrm{N}$ classification (A) and LODDS (B) before IPTW. OS, overall survival; NSCLC, non-small cell lung cancer; LODDS, log odds of positive lymph node; IPTW, inverse probability of treatment weighting. 


\section{Strata + LODDS $<-1.07+-1.07 \leq$ LODDS $<-0.27+$ LODDS $\geq-0.27$}

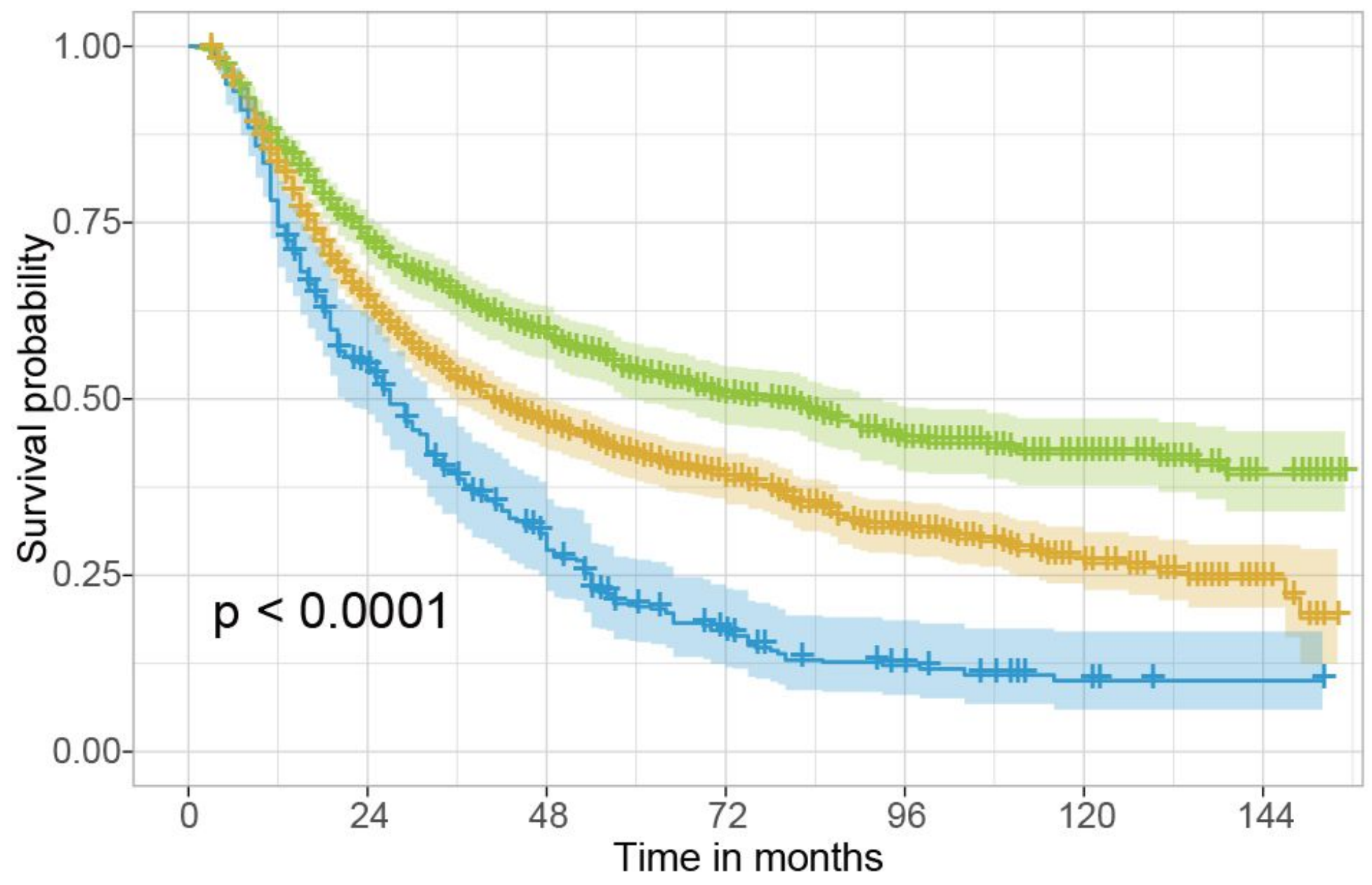

\section{Number at risk}

\begin{tabular}{|c|c|c|c|c|c|c|}
\hline $\begin{array}{ll}\frac{\pi}{\pi} & 1931 \\
\frac{\pi}{2} & 2002 \\
\omega & 1696\end{array}$ & $\begin{array}{l}1249 \\
1166 \\
859\end{array}$ & $\begin{array}{l}759 \\
682 \\
366\end{array}$ & $\begin{array}{l}471 \\
438 \\
169\end{array}$ & $\begin{array}{l}275 \\
236 \\
87\end{array}$ & $\begin{array}{l}170 \\
123 \\
28\end{array}$ & $\begin{array}{l}38 \\
25 \\
9\end{array}$ \\
\hline 0 & 24 & 48 & 72 & 96 & 120 & 144 \\
\hline
\end{tabular}

Figure 3

Kaplan-Meier estimates of OS for NSCLC patients who underwent neoadjuvant therapy stratified by LODDS after IPTW. OS, overall survival; NSCLC, non-small cell lung cancer; LODDS, log odds of positive lymph node; IPTW, inverse probability of treatment weighting. 
A

$\mathrm{ROC}$ at time $\mathrm{t}=1$ year

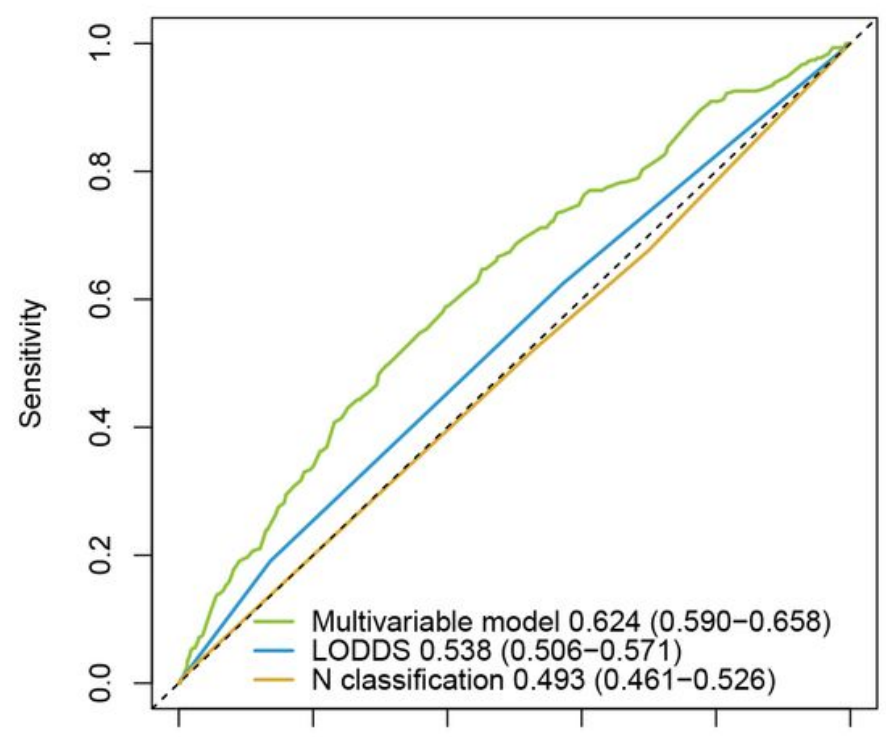

B

\section{$\mathrm{ROC}$ at time $\mathrm{t}=3$ year}

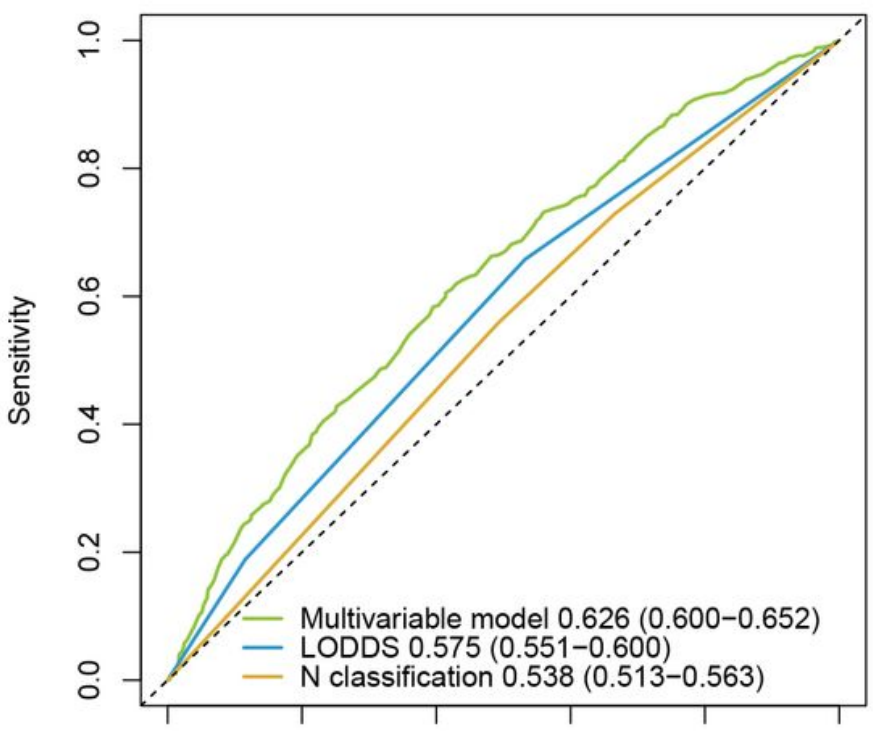

\section{Figure 4}

ROC curves for multivariable model (including LODDS, age, gender, T stage, and radiotherapy), LODDS, and N classification predicting 1-year(A), 3-year (B), 5-year (C), 10-year (D) OS of NSCLC patients underwent neoadjuvant therapy. ROC, receiver operating characteristic; LODDS, log odds of positive lymph node; OS, overall survival; NSCLC, non-small cell lung cancer.

\section{Supplementary Files}


This is a list of supplementary files associated with this preprint. Click to download.

- SupplementaryfileS1.docx 